\title{
Demonstration of the asymmetric lateral Casimir force between corrugated surfaces in the nonadditive regime
}

\author{
H.-C. Chiu, ${ }^{1}$ G. L. Klimchitskaya, ${ }^{2}$ V. N. Marachevsky, ${ }^{3}$ \\ V. M. Mostepanenko, ${ }^{4}$ and U. Mohideen ${ }^{1}$ \\ ${ }^{1}$ Department of Physics and Astronomy, \\ University of California, Riverside, California 92521, USA \\ ${ }^{2}$ North-West Technical University, Millionnaya St. 5, St.Petersburg, 191065, Russia \\ ${ }^{3}$ V.A. Fock Institute of Physics, St.Petersburg \\ State University, St.Petersburg, 198504, Russia \\ ${ }^{4}$ Noncommercial Partnership "Scientific Instruments", \\ Tverskaya St. 11, Moscow, 103905, Russia
}

\begin{abstract}
The measurement of the lateral Casimir force between two aligned sinusoidally corrugated Aucoated surfaces has been performed in the nonadditive regime. The use of deeper corrugations also allowed to demonstrate an asymmetry in the phase dependences of the lateral Casimir force, as predicted earlier. The measurement data are found to be in excellent agreement with the exact theoretical results computed at $\mathrm{T}=300 \mathrm{~K}$ including effect of real material properties. The deviations between the exact theory and the proximity force approximation are quantified. The obtained results are topical for applications in nanomachines.

PACS numbers: 78.20.Ci, 68.35.Af, 68.35.Ct, 85.85.+j
\end{abstract}


The most universally known normal Casimir force [1] leads to an attraction between two closely spaced bodies directed perpendicular to their surfaces. This attraction arises due to zero-point and thermal fluctuations of the electromagnetic field. The phenomenon is important for numerous fields ranging from extra-dimensional physics to nanotechnology and has recently attracted considerable experimental and theoretical attention (see Ref. [2]). The most intriguing feature of the Casimir force is its nonadditivity and thus the complicated dependence on the shape of the boundary surfaces connected with diffraction effects. The nontrivial behavior of the normal Casimir force was experimentally demonstrated in the configuration of a smooth sphere above a sinusoidally corrugated plate [3] in the additive regime. Recently the normal Casimir force between an Au coated sphere and a Si plate with an array of rectangular corrugations was measured in the nonadditive regime [4] (where the corrugation period $\Lambda$ is smaller than the mean separation $z$ between the surfaces [5]). The measurement data were shown to deviate from the additive theoretical results. These deviations, however, are smaller by about $50 \%$ than the prediction of Ref. [6] for ideal metals which might be explained by the interplay between nonzero skin depth and geometry effects [4]. Exact calculations performed at $T=0$ taking into account the nonzero skin depth [7] favor this conjecture. In the configuration of two sinusoidally corrugated bodies (a sphere and a plate) there also arises the lateral Casimir force [8, 9] which gives the possibility to actuate lateral translations in micromachines by means of the electromagnetic zero-point fluctuations. In [9] this force was measured in the additive regime $(\Lambda \gg z)$. It was shown to oscillate sinusoidally as a function of the phase shift between the two corrugations.

In this Rapid Communication we report the first measurement of the lateral Casimir force between two aligned sinusoidally corrugated surfaces of a sphere and a plate in the nonadditive regime where $\Lambda \sim z$. The measurement data are found to be in excellent agreement with the exact computational results at $T=300 \mathrm{~K}$ taking into account real material properties. The deviation of both the experimental data and the exact theory from the prediction of the proximity force approximation (PFA) is quantified. The use here of much deeper corrugations than in Ref. [9] enabled the first demonstration of the asymmetry of the lateral force predicted in [9]. Below we present the obtained experimental results and their comparison with theory by describing only the additional features of this experiment, as compared with Ref. [9]].

The experimental setup is shown in Fig. 1. A chamber with a pressure less than 10 mTorr 
contains the sinusoidally corrugated Au-coated grating of size $5 \times 5 \mathrm{~mm}^{2}$ vertically mounted on the piezotube of an atomic force microscope (AFM). The corrugations have an average period $\Lambda=574.7 \mathrm{~nm}$ (more than two times smaller than in [9] in order to achieve the nonadditive regime) and an amplitude of $A_{1}=85.4 \pm 0.3 \mathrm{~nm}$ (59 nm in [9]). This grating was used as the first test body. A $320 \mu \mathrm{m}$ long V-shaped silicon nitride cantilever of the AFM was uniformly coated with $40 \mathrm{~nm}$ of $\mathrm{Al}$ to improve its thermal and electric conductivity and prevent deformation due to differential thermal expansion in vacuum. Then a $200 \pm$ $4 \mu \mathrm{m}$ diameter polystyrene sphere was placed at the end of the cantilever with conductive Ag epoxy. Next, a freshly cleaved mica sheet of $400 \mu \mathrm{m}$ length, $200 \mu \mathrm{m}$ width and a few micrometer thickness was attached to the bottom of the sphere also with Ag epoxy. One more similar sphere was then attached to the bottom, free end of the mica sheet (see Fig. 1). This last sphere was used as the second test body. The silver epoxy is rigid at all attachments. The resulting system was uniformly coated [10] with a $10 \mathrm{~nm}$ layer of $\mathrm{Cr}$ and then with a $50 \mathrm{~nm}$ layer of $\mathrm{Au}$ in a thermal evaporator.

The lateral Casimir force arises between two perfectly aligned uniaxially corrugated test bodies of the same $\Lambda$ [5, 8, 9]. To imprint corrugations on the sphere, the grating was used as a template. It had a $300 \mathrm{~nm}$ Au coating applied on top of sinusoidal corrugations made of hard epoxy on a $3 \mathrm{~mm}$ thick pyrex substrate. In contrast to Ref. [9] where a soft plastic grating coated with $\mathrm{Al}$ was used, here, the hard epoxy does not require the deposition of the Al layer. The smaller $\Lambda$ required the use of a precise stepper motor and piezo-controlled imprinting technique. At first, the sphere was brought in contact with the grating using a micromanipulator. Then a hard flat surface was moved in $10 \mu \mathrm{m}$ steps using a stepper motor till it comes into contact with the other side of the sphere which is sandwiched against the grating. Next the imprinting was done by applying a voltage to the $z$-piezo to squeeze the sphere between the grating and the hard flat surface. To obtain deeper imprints preserving sphericity additional pressure was put on the sphere using the stepper motor. With the help of the same motor the hard flat surface was gently removed and the sphere was then moved horizontally to a different position on the grating (as the latter might have changed its local amplitude during the imprint process). The amplitude of the imprinted corrugations over a $30 \times 30 \mu \mathrm{m}^{2}$ area on the sphere was measured with an AFM to be $A_{2}=13.7 \pm 0.4 \mathrm{~nm}(60 \%$ increase as compared with [9]). The diameter of the Au-coated sphere was measured to be $2 R=194 \pm 0.3 \mu \mathrm{m}$ using a scanning electron microscope. The corrugations were examined 
using an AFM and found to be homogeneous.

The distinctive feature of the setup shown in Fig. 1 is that a lateral force along the $x$-axis tangential to the corrugated sphere and a grating would lead to the vertical bending of the cantilever (measured using the bi-cell photodiodes A and B in Fig. 1), whereas a force acting normal to the test bodies would lead to a torsional deflection. The torsional spring constant was found to be 46 times larger than the bending spring constant using an independent quad cell AFM measurement of the torsional movement of such a cantilever. Thus, the normal Casimir force could lead to only a negligible change in the second sphere position and the phase of the corrugations. First, the torsional cantilever deflection due to voltages $V$ applied to the grating while the sphere remained grounded was measured by means of the difference signal between the bi-cell photodiodes. The measured deflection signal $S$ at $z=1 \mu \mathrm{m}$, where the Casimir force is negligible, was fitted to the following expression for the normal electrostatic force between the sphere and the grating:

$$
\begin{aligned}
& F_{\text {nor }}^{\mathrm{el}}(z, \varphi)=k_{\mathrm{tor}} S_{\mathrm{nor}}^{\mathrm{el}}=-2 \pi \epsilon_{0}\left(V-V_{0}\right)^{2}\left[\frac{R}{2 z} \frac{1}{\sqrt{1-\beta^{2}}}\right. \\
& +c_{0}+c_{1} \frac{z}{R}+c_{2} \frac{z^{2}\left(2+\beta^{2}\right)}{2 R^{2}}+c_{3} \frac{z^{3}\left(2+3 \beta^{2}\right)}{2 R^{3}} \\
& +c_{4} \frac{z^{4}\left(8+24 \beta^{2}+3 \beta^{4}\right)}{8 R^{4}}+c_{5} \frac{z^{5}\left(8+40 \beta^{2}+15 \beta^{4}\right)}{8 R^{5}} \\
& \left.+c_{6} \frac{z^{6}\left(16+120 \beta^{2}+90 \beta^{4}+5 \beta^{6}\right)}{16 R^{6}}\right] .
\end{aligned}
$$

Here, $k_{\text {tor }}$ is the normal force calibration constant, $V_{0}$ is the residual potential difference between the grating and the sphere when both are grounded, $c_{i}$ are the numbers listed in Ref. [11], $\beta=\left(A_{1}^{2}+A_{2}^{2}-2 A_{1} A_{2} \cos \varphi\right)^{1 / 2} / z$, and $\varphi=2 \pi x / \Lambda$ is the phase shift between the corrugations on both surfaces. Equation (11) was derived using the exact formula for the electric force in the sphere-plate geometry [11] and taking the corrugations into account by means of the PFA. We have checked that within the measurement range, the analytical result (1) coincides up to $2.8 \%$ with the numerical solution of the Poisson equation using the software Comsol Multiphysics (http://www.comsol.com). The good agreement is explained by the absence of diffraction effects for the static electric field. The measurement of $S$ was repeated for eight different voltages between -0.52 to $0.47 \mathrm{~V}$. The mean value of $V_{0}$ found from the fit to Eq. (1) is $V_{0}=-39.6 \pm 1.6 \mathrm{mV}$.

Then the cantilever bending due to the lateral Casimir force was measured. In this case 
only the residual voltage $V_{0}$ was applied to the grating in order to make the electric force equal to zero. The $x$-piezo was used to move the grating along the $x$-axis and thus change $\varphi$. The $z$-piezo, which was independently controlled by an external voltage source, was used to change $z$. The piezo extensions with applied voltage in both directions were calibrated using optical interferometry [12]. Small deviations of the grating from the $x$-axis during the movement were corrected as described in [9]. Initially the corrugated sphere was positioned $3.79 \mathrm{~nm}$ from the separation on contact between the two surfaces $z_{0}$ determined by the corrugations and the highest roughness peaks. The mechanical drift of this position was verified to be very small (average value of $0.14 \mathrm{~nm} / \mathrm{min}$ ). A phase shift was introduced by moving the $x$-piezo continuously for a total distance of $3.3 \mu \mathrm{m}$ at $0.103 \mathrm{~Hz}$. The photodiode signal corresponding to the cantilever deflection was filtered with a low pass filter with a $30 \mathrm{~ms}$ time constant and recorded at each of the 8192 points corresponding to $x$-changes of $0.4 \mathrm{~nm}$. Then the separation from $z_{0}$ was increased by $3.6 \mathrm{~nm}$ to $7.39 \mathrm{~nm}$ and the deflection signal $S_{\text {lat }}^{\mathrm{C}}$ was similarly measured as a function of $\varphi$ and recorded. After this, the separation from $z_{0}$ was increased by $3.96 \mathrm{~nm}$ to $11.35 \mathrm{~nm}$ and the measurements repeated. Next $S_{\text {lat }}^{\mathrm{C}}$ due to the lateral Casimir force as a function of $\varphi$ was measured at separations of 20.05, $32.48,45.30,58.01$ and $70.86 \mathrm{~nm}$ from $z_{0}$.

To convert the measurement data from $S_{\text {lat }}^{\mathrm{C}}$ into values of the lateral Casimir force at some specific separation, it is necessary to determine the values of $k_{\mathrm{ben}}$ and $z_{0}$. This was achieved by measuring the cantilever deflection signal due to the lateral electrostatic force which arises when a voltage is applied to the grating. The measurements of this signal was performed at small separations from close to $z_{0}$ to $z_{0}+120 \mathrm{~nm}$. First, a voltage of $141.456 \mathrm{mV}$ was applied to the grating. The sphere was kept at a distance of $3.96 \mathrm{~nm}$ from $z_{0}$. The phase shift was again changed continuously at a frequency of $0.103 \mathrm{~Hz}$ with the $x$-piezo to a maximum translation of $3.3 \mu \mathrm{m}$. The cantilever deflection corresponding to the total force (sum of the lateral electrostatic and lateral Casimir forces) was recorded as 8192 evenly spaced data points. The sphere was moved further away from the grating by $5.40 \mathrm{~nm}$ to a separation of $9.36 \mathrm{~nm}$ and the measurement was repeated. The cantilever deflection signal due to the total force was measured at many other $z$ at the same voltage and also for a second voltage of $101.202 \mathrm{mV}$ applied to the grating. To obtain the deflection signal due to the lateral electric force alone, the previously measured signal $S_{\text {lat }}^{\mathrm{C}}$ was fitted to the sum of harmonics of the form $A_{k}(z) \sin (k \varphi)$ with $1 \leq k \leq 5$ which takes into account the 
fact that the lateral Casimir force is asymmetric. After determination of the coefficients $A_{k}$, the signal $S_{\text {lat }}^{\mathrm{C}}$ was subtracted from the data for the total deflection signal. As the spheregrating separations in the measurements of the lateral Casimir force and of the total force are not identical, interpolation was used to determine the values of $A_{k}$ at the separations corresponding to the total lateral force. The obtained data for the lateral electrostatic force were fitted to its analytic expression $F_{\text {lat }}^{\mathrm{el}}(z, \varphi)=k_{\text {ben }} S_{\text {lat }}^{\text {el }}$ which is derived from Eq. (1) by the intregration with respect to $z$ and subsequent differentiation with respect to $\varphi$. As a result, the quantities $k_{\mathrm{ben}}$ and $z_{0}$ were found. This was repeated for four different electrostatic force measurements and the average values obtained are $k_{\text {ben }}=1.27 \pm 0.06 \mathrm{nN}$ per unit $S$ and $z_{0}=117.3 \pm 3.0 \mathrm{~nm}$ (see Ref. [13] for details of electrostatic calibrations).

An independent control measurement of $V_{0}$ was done at $z=127.3 \mathrm{~nm}$ using the parabolic dependence of the $F_{\text {lat }}^{\mathrm{el}}$ on $V$. Five different voltages close to the residual potential difference were applied to the grating and the cantilever deflection was measured as a function of $\varphi$ leading to $V_{0}=-39.4 \mathrm{mV}$. This is consistent with $V_{0}=-39.6 \mathrm{mV}$ found earlier at large separations and confirms that the residual potential difference is separation-independent.

The resulting lateral Casimir force $F_{\text {lat }}^{\mathrm{C}}=k_{\mathrm{ben}} S_{\text {lat }}^{\mathrm{C}}$ at a separation $z=124.7 \mathrm{~nm}$ over four corrugation periods is shown in Fig. 2 as dots versus the phase shift $[x / \Lambda=\varphi /(2 \pi)]$ between the corrugated surfaces. Similar results were obtained at all $z$. The mean values and the variances of the $\max F_{\text {lat }}^{\mathrm{C}}$ at each $z$ were calculated using the measurement data for different periods. The maximum values of the $F_{\text {lat }}^{\mathrm{C}}$, as a function of $z$, are shown as crosses in Fig. 3. The arms of the crosses indicate the errors determined at a $95 \%$ confidence level using the procedure of Ref. [11]. The error in the measurement of the absolute separations, $\Delta z$, is a combination of the error in the determination of $z_{0}$ indicated above and half of the first step of the $z$-piezo $\Delta_{p} z \approx 2 \mathrm{~nm}$. By combining these errors, we obtain $\Delta z=4 \mathrm{~nm}$ (a factor of eight improvement as compared with [9]). The errors in the magnitudes of the $F_{\text {lat }}^{\mathrm{C}}$ were found by combining the random errors due to the averaging over the different periods and the systematic errors due to the uncertainty in $k_{\text {ben }}$ (all other systematic errors are negligible). As an example, the total relative error of the $\max F_{\text {lat }}^{\mathrm{C}}$ at $z=121.1 \mathrm{~nm}$ is equal to $24 \%$. At $z=124.7,128.6$, and $137.3 \mathrm{~nm}$ the relative errors are $15 \%, 28 \%$, and $16 \%$, respectively. The nonmonotonous dependence of the error on $z$ is caused by the random error.

The experimental data were compared with computations of the $F_{\text {lat }}^{\mathrm{C}}$ within the scattering approach. The geometry of a perfectly shaped sphere near a flat plate was treated using 
the PFA (this introduces an error of less than $z / R \approx 0.01 \%$ [2]) and the scattering from sinusoidal corrugations was treated precisely using the Rayleigh theory [14] (see also [7]). The dielectric properties of $\mathrm{Au}$ were described by the generalized plasma-like permittivity with the plasma frequency $\omega_{p}=9.0 \mathrm{eV}$ (see [15] for the parameters of oscillators). The influence of the surface roughness on the lateral Casimir force was shown to be negligibly small [9]. The reason is that the rare tall crystals are distributed nonperiodically and do not contribute to the lateral force. The stochastic roughness here has a variance of about $3 \mathrm{~nm}$ and contributes less than $1 \%$ of the force.

The lateral Casimir force between a sinusoidally corrugated grating and a sinusoidally corrugated sphere at $T=300 \mathrm{~K}$ in thermal equilibrium was computed using the formula

$$
\begin{gathered}
F_{\text {lat }}^{C}(z, \varphi)=\frac{4 k_{B} T R}{\Lambda} \int_{z}^{\infty} d z^{\prime} \sum_{l=0}^{\infty} \frac{\partial}{\partial \varphi} \int_{0}^{\infty} d k_{y} \int_{0}^{\pi / \Lambda} d k_{x} \\
\times \ln \operatorname{det}\left[I-R_{1}\left(\boldsymbol{k}_{\perp}, i \xi_{l}\right) R_{2}\left(\boldsymbol{k}_{\perp}, i \xi_{l}, z^{\prime}\right)\right] .
\end{gathered}
$$

Here $k_{B}$ is the Boltzmann constant, $\boldsymbol{k}_{\perp}=\left(k_{x}, k_{y}\right)$ is the wave vector projection on the plane of the grating, $\xi_{l}=2 \pi k_{B} T l / \hbar, l=0,1,2, \ldots$ are the Matsubara frequencies, the prime near the summation sign adds a factor $1 / 2$ to the term with $l=0$, and $R_{1}$ and $R_{2}$ are the reflection matrices from each of the two parallel gratings at the mean separation $z^{\prime}$ (a similar technique was used in [7] for the Casimir energy at $T=0$; alternative techniques for the Casimir energy in nonflat geometries were suggested in [16, 17]). To obtain $R_{1}\left(R_{2}\right)$ one needs the solution of Maxwell equations in the entire space when the other grating is absent. For the corrugated area, Maxwell equations can be rewritten as a system of the first-order ordinary differential equations for the components of the electromagnetic field parallel to the plane of the gratings. In our case of sinusoidal gratings the numerical solution of this system was based on explicit Runge-Kutta formulas of orders 4 and 5, the Dormand-Prince pair, and was performed using the Matlab package. Outside the corrugation area the solutions are given by the Rayleigh expansions. After matching of the solutions in different regions one determines the coefficients of the Rayleigh expansions. Then $R_{1}$ and $R_{2}$ are expressed in terms of these coefficients for the reflection of $E_{y}$ and $H_{y}$ components of the electromagnetic field from the respective gratings. As an example, in Fig. 2 the computational results for $F_{\text {lat }}^{C}$ as a function of $x / \Lambda$ at $z=124.7 \mathrm{~nm}$ are shown as a solid line which is in a very good agreement with the data with no fitting parameters. Both the dots and the theoretical line demonstrate that the lateral Casimir force is asymmetric (the dependence of the $F_{\text {lat }}^{C}$ 
on $\varphi$ is strictly sinusoidal only if the calculation is restricted to the lowest order $\sim A_{1} A_{2}$ [5, 9]). The true asymmetry of the lateral force is obvious even without the theory curve because the average shift of each maximum point from the midpoint of two adjacent minima is $(0.12 \pm 0.02) \Lambda$.

The computational results for the maximum value of $F_{\text {lat }}^{C}$ versus $z$ are shown in Fig. 3 as the solid line which is in an excellent agreement with the experimental data. For comparison purposes the dashed line in Fig. 3 shows the maximum values of $F_{\text {lat }}^{C}$ computed using the PFA applied not only to the sphere-plate configuration [as in Eq. (2)] but to sinusoidal corrugations as well. To find the Casimir energy between two corrugated gratings, we have used the standard Lifshitz formula which includes the skin depth effects, but replaced separation $z$ with $z+A_{2} \sin \left(2 \pi x^{\prime} / \Lambda+\varphi\right)-A_{1} \sin \left(2 \pi x^{\prime} / \Lambda\right)$ and made an averaging over $x^{\prime}$. As can be seen in Fig. 3, the PFA force amplitudes are significantly larger than both the experimental data and the exact computational results. The deviation increases from about $18 \%$ at $z=120 \mathrm{~nm}$ to $60 \%$ at $z=180 \mathrm{~nm}$. This is explained by the fact that for the shorter $\Lambda$ used in this work the additive approaches are not applicable [5, 6].

To conclude, we have performed the measurement of the lateral Casimir force between sinusoidally corrugated surfaces of small period and compared the obtained data with the recently developed exact theory taking into account real material properties of the boundary metal. This has allowed the first demonstration of the asymmetry of the lateral Casimir force and has permitted to quantify deviations from the PFA. The obtained results enhance the capabilities for the application of the Casimir effect in nanotechnology, specifically for frictionless transmission of lateral motion [18, 19], and achieve a better ubderstanding of dispersion forces between nonplanar boundaries.

This work was supported by the NSF Grant No. PHY0653657 (measurement of the lateral Casimir force) and DOE Grant No. DE-FG02-04ER46131 (calculation of the electric and Casimir forces). G.L.K. and V.M.M. were also supported by the DFG Grant GE 696/9-1. V.N.M. was supported by the Grants RNP 2.1.1/1575 and RFBR 07-01-00692-a.

[1] H. B. G. Casimir, Proc. K. Ned. Akad. Wet. B 51, 793 (1948).

[2] G. L. Klimchitskaya, U. Mohideen, and V. M. Mostepanenko, arXiv:0902.4022, Rev. Mod. 
Phys. (to be published); 60 Years of the Casimir Effect, Eds. G. Barton, G. Carugno, V. V. Dodonov, and M. Man'ko, J. Phys.: Conf. Ser. 161 (2009).

[3] A. Roy and U. Mohideen, Phys. Rev. Lett. 82, 4380 (1999).

[4] H. B. Chan et al., Phys. Rev. Lett. 101, 030401 (2008).

[5] T. Emig et al., Phys. Rev. A 67, 022114 (2003).

[6] R. Büscher and T. Emig, Phys. Rev. A 69, 062101 (2004).

[7] A. Lambrecht and V. N. Marachevsky, Phys. Rev. Lett. 101, 160403 (2008); Int. J. Mod. Phys. A 24, 1789 (2009).

[8] R. Golestanian and M. Kardar, Phys. Rev. Lett. 78, 3421 (1997).

[9] F. Chen, U. Mohideen, G. L. Klimchitskaya, and V. M. Mostepanenko, Phys. Rev. Lett. 88, 101801 (2002); Phys. Rev. A 66, 032113 (2002).

[10] H.-C. Chiu, C.-C. Chang, R. Castillo-Garza, F. Chen, and U. Mohideen, J. Phys. A 41, 164022 (2008).

[11] F. Chen, U. Mohideen, G. L. Klimchitskaya, and V. M. Mostepanenko, Phys. Rev. A 74, $022103(2006)$.

[12] F. Chen and U. Mohideen, Rev. Sci. Instrum. 72, 3100 (2001).

[13] M. Bordag, G. L. Klimchitskaya, U. Mohideen, and V. M. Mostepanenko, Advances in the Casimir Effect (Oxford University Press, Oxford, 2009).

[14] O. M. Rayleigh, Proc. Roy. Soc. A 79, 399 (1907).

[15] R. S. Decca, D. López, E. Fischbach, G. L. Klimchitskaya, D. E. Krause, and V. M. Mostepanenko, Eur. Phys. J. C 51, 963 (2007).

[16] T. Emig, N, Graham, R. L. Jaffe, and M. Kardar, Phys. Rev. Lett. 99, 170403 (2007).

[17] O. Kenneth and I. Klich, Phys. Rev. B 78, 014103 (2008).

[18] A. Ashourvan, M. F. Miri, and R. Golestanian, Phys. Rev. Lett. 98, 140801 (2007).

[19] T. Emig, Phys. Rev. Lett. 98, 160801 (2007). 
FIG. 1: (Color online) Schematic of the experimental setup (see text for further details). Insertion shows the imprinted corrugations on the second sphere. The lighter color shows higher points and hence demonstrates the sphericity of the imprinted surface.

FIG. 2: (Color online) The phase dependence of the lateral Casimir force. The measurement data are shown as dots. The solid line is the exact theory.

FIG. 3: The maximum values of the measured lateral Casimir force are shown as crosses. The solid and dashed lines are the predictions of the exact theory and the PFA, respectively. 
This figure "figLF-1.gif" is available in "gif" format from: http://arxiv.org/ps/0909.2161v1 
This figure "figLF-2.gif" is available in "gif" format from: http://arxiv.org/ps/0909.2161v1 
This figure "figLF-3.gif" is available in "gif" format from: http://arxiv.org/ps/0909.2161v1 